\title{
Formation control with disturbance rejection for a class of Lipschitz nonlinear systems
}

DOI:

10.1007/s11432-016-9125-2

\section{Document Version}

Accepted author manuscript

Link to publication record in Manchester Research Explorer

\section{Citation for published version (APA):}

Wang, C., Zuo, Z., Gong, Q., \& Ding, Z. (2017). Formation control with disturbance rejection for a class of Lipschitz nonlinear systems. Science China. Information Sciences, 60(7), [070202]. https://doi.org/10.1007/s11432-0169125-2

\section{Published in:}

Science China. Information Sciences

\section{Citing this paper}

Please note that where the full-text provided on Manchester Research Explorer is the Author Accepted Manuscript or Proof version this may differ from the final Published version. If citing, it is advised that you check and use the publisher's definitive version.

\section{General rights}

Copyright and moral rights for the publications made accessible in the Research Explorer are retained by the authors and/or other copyright owners and it is a condition of accessing publications that users recognise and abide by the legal requirements associated with these rights.

\section{Takedown policy}

If you believe that this document breaches copyright please refer to the University of Manchester's Takedown Procedures [http://man.ac.uk/04Y6Bo] or contact uml.scholarlycommunications@manchester.ac.uk providing relevant details, so we can investigate your claim.

\section{OPEN ACCESS}




\title{
Formation Control with Disturbance Rejection for a Class of Lipschitz Nonlinear Systems
}

\author{
Chunyan Wang ${ }^{1,4}$, Zongyu Zuo², Qinghai Gong ${ }^{3,4} \&$ Zhengtao Ding $^{1,4 *}$ \\ ${ }^{1}$ School of Electrical and Electronic Engineering, University of Manchester, Sackville Street Building, \\ Manchester M13 9PL, UK (chunyan.wang-2@manchester.ac.uk, zhengtao.ding@manchester.ac.uk); \\ ${ }^{2}$ The Seventh Research Division, and Science and Technology on Aircraft Control Laboratory, Beihang University, \\ Beijing 100191, China (zzybobby@buaa.edu.cn); \\ ${ }^{3}$ Beijing Aerospace Automatic Control Institute, P.O.Box 142-402, Beijing, 100854, China (qinghaigong@126.com); \\ ${ }^{4}$ Sino-British Joint Advanced Control System Technology Laboratory, James Lighthill Building, Manchester, M13 9PL, UK
}

\begin{abstract}
In this paper, we consider the leader-follower formation control problem for general multi-agent systems with Lipschitz nonlinearity and unknown disturbances. To deal with the disturbances, a disturbance observer-based control strategy is developed for each follower. Then, a time-varying formation protocol is proposed based on the relative state of the neighbouring agents and sufficient conditions for global stability of the formation control are identified using Lyapunov method in the time domain. The proposed strategy and analysis guarantee that all signals in the closed-loop dynamics are uniformly ultimately bounded and the formation tracking error converges to an arbitrarily small residual set. Finally, the validity of the proposed controller is demonstrated through a numerical example.
\end{abstract}

Keywords Multi-agent systems, formation control, Lipschitz nonlinearity, disturbance rejection

\section{Introduction}

In recent years, cooperative control of a group of agents has been extensively researched due to its high potential in many applications, such as cooperative monitoring, surveillance, rural search and rescue. Different strategies, including centralized, decentralized, and distributed ones, have been used for controlling such systems 1. Compared with centralized systems, multi-agent systems with distributed control strategy have preferred flexibility and robustness. By using local neighbor-to-neighbor interaction, distributed multi-agent systems can reduce the signal communication and computational workload efficiently. Consensus control is one of the most important topics in distributed cooperative control and many theoretic and practical issues have been analysed and reported in literature, $1-7$, to cite a few. Theoretical explanation is given in 2 to prove that the nearest neighbour rule can force the agents to stay together and move in the same direction without centralized coordination. A general consensus framework is given in 3 for a group agents with integrator-type dynamics. Since then, consensus problems have been intensively studied in different directions in literature $8-14$. The results for consensus control can be divided into leader-follower type consensus (also named consensus tracking), which has a reference consensus value, and leaderless consensus, where the consensus value is depended on the connections and initial conditions of the agents.

\footnotetext{
*Corresponding author (email: zhengtao.ding@manchester.ac.uk)
} 
Formation control is one of the most important applications in cooperative control. Different control strategies have been used for formation control, such as actual leader |15, virtual leader 16], behavioural 17, etc. A survey on various classifications of formation control is given in 18 . Consensus based formation control strategies have also been investigated $19-25$. The role of Laplacian eigenvalues in determining formation stability is investigated in $\mid 19$. In $20 \mid$, it is pointed out that consensus based formation control strategies are more general and contain many existing actual/virtual leader and behavioural approaches as special cases. Most of the early results on formation control focus on simple agent dynamics such as first or second-order integrator dynamics. In reality, some practical physical systems cannot be feedback linearised as first or second-order dynamical model. Formation control for a class of high-order linear multi-agent systems with time delays are studied in 22 . The applications of formation control in various areas could be found in $23-25$. It is worth noting that only linear dynamics are considered in the above results.

In many scenarios, external disturbances are unavoidable and will degrade the performance of the designed systems. In extreme cases, external disturbances will induce the loss of stability of the closed-loop systems. Many control methods have been used to deal with different external disturbances. For systems with bounded external disturbances, $H_{\infty}$ control method is reliable and has been extensively investigated in literature $26-28$. For some periodic disturbances which have inherent characteristics such as harmonics and unknown constant load, disturbance observer can be designed based on the state/output information and then the disturbance estimate can be used in the design of control input to cancel the disturbances directly $\mid 29$. Typical results for disturbance observer design may be found in the references $29-34$. Output regulation design is also an efficient way to deal with deterministic disturbances. Many results have been published in this area for single systems 35,36 and multi-agent systems 37-40.

In this paper, we consider the time-varying formation problem for Lipschitz nonlinear multi-agent systems subject to external disturbances. Compared with the previous works, the key features of this paper are as follows: (i) the time-varying formation problem is considered for general systems with directed topology; (ii) external disturbances with unknown amplitudes and phases are considered in the formation control design; (iii) the influence of the Lipschitz nonlinearity is taken into account in the stability analysis. A disturbance observer-based control strategy is developed and sufficient conditions for global stability of the formation problem are derived with Lyapunov method. The proposed strategy and analysis guarantee that the formation tracking errors are uniformly ultimately bounded for any initial conditions. A numerical example is provided to demonstrate the proposed design.

The remainder of this paper is organized as follows. In Section 2 , the problem formulation is introduced. Section 3 presents some notations and preliminary results. Section 4 presents the main results on the formation control and disturbance rejection. Simulation results are given in Section 5. Section 6 concludes the paper.

\section{Problem Statement}

Consider a group of $N+1$ agents, consisting of $N$ followers and one leader indexed by 0 :

$$
\dot{x}_{i}(t)=A x_{i}(t)+B u_{i}(t)+\phi\left(x_{i}(t)\right)+B F \omega_{i}(t),
$$

where for agent $i, i=0,1, \ldots, N, x_{i} \in \mathbb{R}^{n}$ is the state, $u_{i} \in \mathbb{R}^{m}$ is the control input, $A \in \mathbb{R}^{n \times n}, B \in \mathbb{R}^{n \times m}$ and $F \in \mathbb{R}^{m \times s}$ are constant matrices with $(A, B)$ being controllable, the unknown nonlinear function $\phi: \mathbb{R}^{n} \rightarrow \mathbb{R}^{n}, \phi(0)=0$, is assumed to satisfy the Lipschitz condition as

$$
\|\phi(\alpha)-\phi(\beta)\| \leqslant \gamma\|\alpha-\beta\|, \forall \alpha, \beta \in \mathbb{R}^{n}
$$

where $\gamma>0$ is the Lipschitz constant, and $\omega_{i} \in \mathbb{R}^{s}$ is a disturbance that is generated by a linear exogenous system

$$
\dot{\omega}_{i}(t)=S \omega_{i}(t)
$$


with $S \in \mathbb{R}^{s \times s}$ is known and $(S, B F)$ is observable. For the leader-follower structure, it is reasonable to assume that the leader has no neighbours, and the leader's control input is zero, i.e. $u_{0}=0, \omega_{0}=0$.

The objective of this paper is to design a distributed formation protocol for each follower, such that the formation tracking errors $e_{i}(t)=x_{i}(t)-d_{i}(t)-x_{0}(t)$ are uniformly ultimately bounded for any initial condition $x_{i}(0), i=0,1, \cdots, N$, where $d_{i}(t)$ is a specified time-varying formation pattern between the leader and the $i$ th follower. For the convenience, we define $d_{0}(t) \equiv 0, e_{0}(t) \equiv 0$.

Assumption 1. The eigenvalues of $S$ are distinct, and lie on the imaginary axis.

Assumption 2. The specified formation vector $d(t)=\left[d_{1}^{T}(t), d_{1}^{T}(t), \cdots, d_{N}^{T}(t)\right]^{T}$ is bounded with $d_{i}(t), \forall i=1,2, \cdots, N$ continuously differentiable, i.e., $\|d(t)\| \leqslant \epsilon_{0}$, where $\epsilon_{0}$ is a positive constant.

Assumption 3. The $i$ th agent can obtains its neighbors' formation information via the inter-agent communication.

Remark 1. Assumption 1 is commonly used in output rejection and disturbance rejection design 29,35 . System (2) can represents unknown constant disturbances, or sinusoidal functions with unknown amplitudes and phases, or some other functions which can be approximated by sinusoidal functions with a bias. Assumption 3 does not imply loss of generality because there are always communications between the $i$ th agent and its neighbors.

\section{Preliminary Results}

\subsection{Notations}

In this paper, let $I_{N}$ and $\mathbf{1}$ denote the identity matrix of dimension $N$ and a column vector with all entries equal to one, respectively. Let $\mathbb{R}^{n \times m}$ represent a set of $n \times m$ real matrices, and $0_{n \times m}$ denote the matrix with all zeros. Given a real vector $x \in \mathbb{R}^{n},\|x\|$ is the Euclidean norm of $x$. For a matrix $P, \lambda_{\min }(P)$ and $\lambda_{\max }(P)$ represent its minimum and maximum eigenvalue, respectively. Given two matrices $X$ and $Y, X \otimes Y$ denotes the Kronecker product of the matrices. $\operatorname{diag}\left(A_{i}\right)$ denotes a block-diagonal matrix with $A_{i}, i=1, \cdots, N$, on the diagonal entry. Given two symmetric real matrices $A$ and $B, A>B$ denotes that $A-B$ is positive definite.

\subsection{Graph theory}

The communication connections among agents are described by a graph $\mathcal{G}(\mathcal{V}, \mathcal{E})$, where $\mathcal{V}$ represent the agents and $\mathcal{E}$ represent the connections between the agents. In the directed graph, $(i, j) \in \mathcal{E}$ represents the communication from the $i$ th agent to the $j$ th agent, but not vice versa. In the undirected graph, $(i, j) \in \mathcal{E}$ means that the information can be exchanged between the $i$ th agent and $j$ th agent. For $N+1$ agents, the associated adjacency matrix of $\mathcal{G}$ is defined as $\mathcal{A}=\left[a_{i j}\right]_{(N+1) \times(N+1)} \in \mathbb{R}^{(N+1) \times(N+1)}$. If there is a connection from agent $j$ to agent $i, a_{i j}=1$; otherwise $a_{i j}=0$. The Laplacian matrix $\mathcal{L}=\left[l_{i j}\right]_{(N+1) \times(N+1)}$ associated with $\mathcal{A}$ is defined by $l_{i i}=\sum_{j=1}^{N+1} a_{i j}$ and $l_{i j}=-a_{i j}$ when $i \neq j$.

Assumption 4. The communication topology $\mathcal{G}$ contains a directed spanning tree with the leader as the root.

With Assumption 4, we know that the Laplacian matrix $\mathcal{L}$ of the communication topology $\mathcal{G}$ has simple zero eigenvalue with right eigenvector $\mathbf{1}=[1,1, \ldots, 1]^{T}$ and all the other eigenvalues of $\mathcal{L}$ have positive real parts 7 . Since the leader has no neighbours, the Laplacian matrix $\mathcal{L}$ has the following structure

$$
\mathcal{L}=\left[\begin{array}{cc}
0 & 0_{1 \times N} \\
\mathcal{L}_{2} & \mathcal{L}_{1}
\end{array}\right],
$$

where $\mathcal{L}_{1} \in \mathbb{R}^{N \times N}$ and $\mathcal{L}_{2} \in \mathbb{R}^{N \times 1}$. It can be seen that $\mathcal{L}_{1}$ is a nonsingular M-matrix. We also have the following result for $\mathcal{L}_{1}$ : 
Lemma 1. 23 For the nonsingular M-matrix $\mathcal{L}_{1}$, there exists a positive diagonal matrix $Q$ such that

$$
Q \mathcal{L}_{1}+\mathcal{L}_{1}^{T} Q \geqslant \rho_{0} I
$$

for some positive constant $\rho_{0}$. $Q$ can be constructed by letting $Q=\operatorname{diag}\left\{q_{1}, q_{2}, \cdots, q_{N}\right\}$, where $q=$ $\left[q_{1}, q_{2}, \cdots, q_{N}\right]^{T}=\left(\mathcal{L}_{1}^{T}\right)^{-1}[1,1, \cdots, 1]^{T}$.

\subsection{Preliminary Results}

Lemma 2. 41 For a given continuous system $\dot{z}=y(z, t), y(\cdot)$ is assumed locally Lipschitz in $z$. If there exists a differentiable function $V(z, t) \geqslant 0$ such that

$$
\begin{aligned}
& \beta_{1}(\|z\|) \leqslant V(z, t) \leqslant \beta_{2}(\|z\|), \\
& \dot{V}(z, t) \leqslant-\beta_{3}(\|z\|)+\iota,
\end{aligned}
$$

where $\iota>0$ is a constant, $\beta_{1}, \beta_{2}$ belong to class $\mathcal{K}_{\infty}$ functions, and $\beta_{3}$ belongs to class $\mathcal{K}$ function. The solution $z(t)$ of the system $\dot{z}=y(z, t)$ is uniformly ultimately bounded.

Lemma 3 (Young's Inequality). For nonnegative real numbers $a, b$, if $p, q$ are real numbers that satisfy $\frac{1}{p}+\frac{1}{q}=1$, then $a b \leqslant \frac{a^{p}}{p}+\frac{b^{q}}{q}$.

Lemma 4 (Schur Complement Lemma). For any constant symmetric matrix

$$
S=\left[\begin{array}{ll}
S_{11} & S_{12} \\
S_{12} & S_{22}
\end{array}\right],
$$

the following statements are equivalent:

(1) $S<0$,

(2) $S_{11}<0, S_{22}-S_{12}^{T} S_{11}^{-1} S_{12}<0$,

(3) $S_{22}<0, S_{11}-S_{12} S_{22}^{-1} S_{12}^{T}<0$.

\section{Main results}

With the formation tracking errors $e_{i}(t)=x_{i}(t)-d_{i}(t)-x_{0}(t)$, we have

$$
\dot{e}_{i}(t)=A e_{i}(t)+B u_{i}(t)+\varepsilon_{i}(t)+B F \omega_{i}(t)+A d_{i}(t)-\dot{d}_{i}(t),
$$

where $\varepsilon_{i}(t)=\phi\left(x_{i}\right)-\phi\left(x_{0}\right)$. Define $e(t)=\left[e_{1}^{T}(t), e_{2}^{T}(t), \cdots, e_{N}^{T}(t)\right]^{T}$. Then, the leader-follower formation of system (1) is achieved when $\lim _{t \rightarrow \infty} e(t)=0$, as $e=0$ implies that $x_{0}=x_{1}-d_{1}=\cdots=x_{N}-d_{N}$. Therefore, the formation problem of system (1) is transformed into the regulator problem of system (4).

With the relative state of the neighbouring agent

$$
\begin{aligned}
z_{i}(t) & =\sum_{j=0}^{N} a_{i j}\left[\left(x_{i}(t)-d_{i}(t)\right)-\left(x_{j}(t)-d_{j}(t)\right)\right] \\
& =\sum_{j=0}^{N} a_{i j}\left[e_{i}(t)-e_{j}(t)\right] \\
& =\sum_{j=1}^{N} a_{i j}\left[e_{i}(t)-e_{j}(t)\right]+a_{i 0} e_{i}(t) \\
& =\sum_{j=1}^{N} l_{i j} e_{j}(t),
\end{aligned}
$$

a formation controller is proposed as

$$
u_{i}(t)=c K z_{i}+v_{i}(t)-F \hat{\omega}_{i},
$$


where $c \geqslant 2 q_{\max } / \rho_{0}$ is a positive real constant with $q_{\max }=\max \left\{q_{1}, q_{2}, \cdots, q_{N}\right\}, v_{i}(t) \in \mathbb{R}^{m}$ is the external command input, $\hat{\omega}_{i}(t)$ is generated by the following disturbance observer:

$$
\left\{\begin{array}{l}
\hat{\omega}_{i}=\eta_{i}+L z_{i} \\
\dot{\eta}_{i}=S \eta_{i}+(S L-L A) z_{i}-c L B K \sum_{j=1}^{N} l_{i j} z_{j}
\end{array}\right.
$$

where $K \in \mathbb{R}^{m \times n}$ and $L \in \mathbb{R}^{s \times n}$ are the control and the observer gain to be designed later.

By (5), the closed-loop error dynamics of the $i$ th agent can be obtained as

$$
\dot{e}_{i}(t)=A e_{i}(t)+c B K \sum_{j=1}^{N} l_{i j} e_{j}(t)+\varepsilon_{i}(t)-B F \tilde{\omega}_{i}(t)+B v_{i}(t)+A d_{i}(t)-\dot{d}_{i}(t),
$$

where $\tilde{\omega}_{i}(t)=\hat{\omega}_{i}-\omega_{i}$. Therefore, the formation of systems (1) is achieved if the following systems

$$
\dot{e}_{i}(t)=A e_{i}(t)+c B K \sum_{j=1}^{N} l_{i j} e_{j}(t)+\varepsilon_{i}(t)-B F \tilde{\omega}_{i}(t),
$$

are asymptotically stable, and

$$
\lim _{t \rightarrow \infty}\left(B v_{i}(t)+A d_{i}(t)-\dot{d}_{i}(t)\right)=0
$$

$\forall i=1,2, \cdots, N$.

With the controller and observer shown in (5) and (6), $K$ and $L$ are chosen as

$$
\begin{aligned}
K & =-B^{T} P, \\
L & =\rho_{1} M^{-1}(B F)^{T},
\end{aligned}
$$

where $P>0, M>0$ are constant matrices to be designed, $\rho_{1}$ is a scaler.

Theorem 1. For systems (8), the robust stability problem with disturbance rejection can be solved by the control algorithm (5) with (10)-(11) if there exist positive definite matrices $P, M$ and constants $\rho_{1}, \kappa>0$, such that

$$
\left[\begin{array}{cc}
A W+W A^{T}-2 B B^{T}+2 \kappa I & W \\
W & -\frac{q_{\min }}{2 \gamma^{2} r_{1}+\kappa_{1}}
\end{array}\right]<0,
$$

are satisfied with $W=P^{-1}, r_{1}=q_{\max }\left(\rho_{1} \sigma_{\max }^{2}\left(\mathcal{L}_{1}\right)+1\right) / \kappa, r_{2}=2 \rho_{1} / c-q_{\max } / \kappa q_{\min }^{-1}, q_{\min }=\min \left\{q_{1}, q_{2}, \cdots, q_{N}\right\}$, $q_{\max }=\max \left\{q_{1}, q_{2}, \cdots, q_{N}\right\}, \kappa_{1}$ is any positive number.

Proof. Systems (8) can be written in the compact form as

$$
\dot{e}(t)=\left(I_{N} \otimes A\right) e(t)+c\left(\mathcal{L}_{1} \otimes B K\right) e(t)+\varepsilon(t)-\left(I_{N} \otimes B F\right) \tilde{\omega}(t),
$$

where $\varepsilon=\left[\varepsilon_{1}^{T}, \varepsilon_{2}^{T}, \cdots, \varepsilon_{N}^{T}\right]^{T}, \tilde{\omega}=\left[\tilde{\omega}_{1}^{T}, \tilde{\omega}_{2}^{T}, \cdots, \tilde{\omega}_{N}^{T}\right]^{T}$. For disturbance observer error $\tilde{\omega}_{i}(t)$, with condition (9), a direct differentiation gives that

$$
\begin{aligned}
\dot{\tilde{\omega}}_{i}(t) & =\dot{\eta}_{i}(t)+L \sum_{j=1}^{N} l_{i j} \dot{e}_{j}(t)-S \omega_{i}(t) \\
& =S \tilde{\omega}_{i}(t)-L B F \sum_{j=1}^{N} l_{i j} \tilde{\omega}_{j}(t)+L \sum_{j=1}^{N} l_{i j} \varepsilon_{j},
\end{aligned}
$$

which can be written in the compact form as

$$
\dot{\tilde{\omega}}(t)=\left(I_{N} \otimes S\right) \tilde{\omega}(t)-\left(\mathcal{L}_{1} \otimes L B F\right) \tilde{\omega}(t)+\left(\mathcal{L}_{1} \otimes L\right) \varepsilon .
$$


Consider the Lyapunov function candidate

$$
V(t)=e^{T}(t)(Q \otimes P) e(t)+\tilde{\omega}^{T}(t)(Q \otimes M) \tilde{\omega}(t) .
$$

The derivative of $V(t)$ along the trajectory of (14) and (15) can be obtained as

$$
\begin{aligned}
\dot{V}(t)= & e^{T}(t)\left(Q \otimes\left(P A+A^{T} P\right)-c\left(Q \mathcal{L}_{1}+\mathcal{L}_{1}^{T} Q\right) \otimes P B B^{T} P\right) e(t)+2 \sum_{i=2}^{N} q_{i} e_{i}^{T}(t) P\left(\varepsilon_{i}-B F \tilde{\omega}_{i}(t)\right) \\
& +\tilde{\omega}^{T}(t)\left(Q \otimes\left(M S+S^{T} M\right)-\rho_{1}\left(Q \mathcal{L}_{1}+\mathcal{L}_{1}^{T} Q\right) \otimes F^{T} B^{T} B F\right) \tilde{\omega}(t)-2 \rho_{1} \tilde{\omega}^{T}(t)\left(Q \mathcal{L}_{1} \otimes(B F)^{T}\right) \varepsilon \\
\leqslant & e^{T}(t)\left(Q \otimes\left(P A+A^{T} P\right)-c \rho_{0} I \otimes P B B^{T} P\right) e(t)+2 \sum_{i=2}^{N} q_{i} e_{i}^{T}(t) P\left(\varepsilon_{i}-B F \tilde{\omega}_{i}(t)\right) \\
& +\tilde{\omega}^{T}(t)\left(Q \otimes\left(M S+S^{T} M\right)-\rho_{1} \rho_{0} I \otimes F^{T} B^{T} B F\right) \tilde{\omega}(t)-2 \rho_{1} \tilde{\omega}^{T}(t)\left(Q \mathcal{L}_{1} \otimes(B F)^{T}\right) \varepsilon \\
\leqslant & e^{T}(t)\left(Q \otimes\left(P A+A^{T} P+2 \kappa P P\right)-c \rho_{0} I \otimes P B B^{T} P\right) e(t)+\frac{q_{\max }}{\kappa}\|\varepsilon\|^{2}-2 \rho_{1} \tilde{\omega}^{T}(t)\left(Q \mathcal{L}_{1} \otimes(B F)^{T}\right) \varepsilon \\
& +\tilde{\omega}^{T}(t)\left(Q \otimes\left(M S+S^{T} M+\frac{q_{\max }}{\kappa q_{\min }} F^{T} B^{T} B F\right)-\rho_{1} \rho_{0} I \otimes F^{T} B^{T} B F\right) \tilde{\omega}(t) \\
\leqslant & e^{T}(t)\left(Q \otimes\left(P A+A^{T} P+2 \kappa P P-2 P B B^{T} P\right)\right) e(t)+r_{1}\|\varepsilon\|^{2} \\
& +\tilde{\omega}^{T}(t)\left(Q \otimes\left(M S+S^{T} M-r_{2} F^{T} B^{T} B F+r_{3} I\right)\right) \tilde{\omega}(t),
\end{aligned}
$$

where $r_{1}=q_{\max }\left(\rho_{1} \sigma_{\max }^{2}\left(\mathcal{L}_{1}\right)+1\right) / \kappa, r_{2}=2 \rho_{1} / c-q_{\max } / \kappa q_{\min }^{-1}, r_{3}=q_{\max } \rho_{1} \kappa \lambda_{\max }\left(F^{T} B^{T} B F\right) / q_{\min }$.

The nonlinear term $\varepsilon(x)$ in the new system (7) is related to state $x(t)$. For the formation stability analysis, a bound of this term needs to be found in terms of the transformed state $e(t)$.

Lemma 5. For the nonlinear function $\varepsilon(x)$ in (7), a bound can be established in terms of the state $e(t)$ as

$$
\|\varepsilon\|^{2} \leqslant 2 \gamma^{2}\left(\|e(t)\|^{2}+\|d(t)\|^{2}\right)
$$

Proof. Based on the state transformation $e_{i}(t)=x_{i}(t)-d_{i}(t)-x_{0}(t)$, and $\varepsilon_{i}=\phi\left(x_{i}\right)-\phi\left(x_{0}\right)$, we have

$$
\left\|\varepsilon_{i}\right\|=\left\|\phi\left(x_{i}\right)-\phi\left(x_{0}\right)\right\| \leqslant \gamma\left\|x_{i}-x_{0}\right\| .
$$

It then follows that

$$
\left\|\varepsilon_{i}\right\| \leqslant \gamma\left\|e_{i}(t)+d_{i}(t)\right\| \leqslant \gamma\left(\left\|e_{i}(t)\right\|+\left\|d_{i}(t)\right\|\right),
$$

and

$$
\|\varepsilon\|^{2}=\sum_{i=1}^{N}\left\|\varepsilon_{i}\right\|^{2} \leqslant 2 \gamma^{2} \sum_{i=1}^{N}\left(\left\|e_{i}(t)\right\|^{2}+\left\|d_{i}(t)\right\|^{2}\right)=2 \gamma^{2}\left(\|e(t)\|^{2}+\|d(t)\|^{2}\right),
$$

where we have used $\sum_{k=1}^{N}\left\|e_{i}\right\|^{2}=\|e\|^{2}$ and the inequality $(a+b)^{2} \leqslant 2\left(a^{2}+b^{2}\right)$. This completes the proof.

From (18) and (19), we obtain that

$$
\begin{aligned}
\dot{V}(t) \leqslant & e^{T}(t)\left(Q \otimes\left(P A+A^{T} P+2 \kappa P P-2 P B B^{T} P+2 \gamma^{2} r_{1} / q_{\min } I\right)\right) e(t) \\
& +\tilde{\omega}^{T}(t)\left(Q \otimes\left(M S+S^{T} M-r_{2} F^{T} B^{T} B F+r_{3} I\right)\right) \tilde{\omega}(t)+2 \gamma^{2} r_{1}\|d(t)\|^{2} \\
\leqslant & e^{T}(t)\left(Q \otimes P_{1}\right) e(t)+\tilde{\omega}^{T}(t)\left(Q \otimes M_{1}\right) \tilde{\omega}(t)+2 \gamma^{2} r_{1}\|d(t)\|^{2},
\end{aligned}
$$

where

$$
\begin{aligned}
P_{1} & =P A+A^{T} P+2 \kappa P P-2 P B B^{T} P+2 \gamma^{2} r_{1} / q_{\min } I, \\
M_{1} & =M S+S^{T} M-r_{2} F^{T} B^{T} B F+r_{3} I .
\end{aligned}
$$




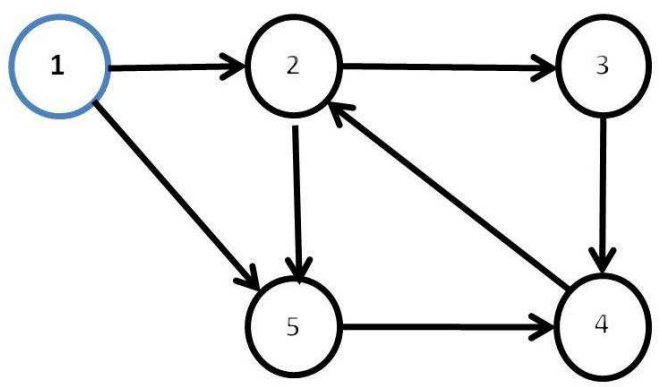

Figure 1 Communication topology.

With (24) and (25), it can be shown by Schur Complement that conditions (12) and (13) are respectively equivalent to $P_{1}<-\kappa_{1} / q_{\min } I$ and $M_{1}<-\kappa_{1} / q_{\min } I$, which further implies from (23) that $\dot{V}(t)<$ $-\kappa_{1}\left(\|e(t)\|^{2}+\|\tilde{\omega}(t)\|^{2}\right)+2 \gamma^{2} r_{1}\|d(t)\|^{2}$. Since $d(t)$ is bounded as mentioned in Assumption 2, the positive term could be very small by choosing appropriate free scaler $\kappa$. By Lemma 2 , we have that the tracking error $e(t)$ is uniformly ultimately bounded and the disturbance rejection is achieved.

Remark 2. The state transformation can only apply to the linear parts, and the nonlinear functions remain functions of the original state, which leads to extra complexity in the stability analysis. Due to the nonlinear terms, the formation tracking errors can only be uniformly ultimately bounded instead of converging to zero. If the Lipschitz constant $\gamma \equiv 0$, the formation tracking errors will converge to zero and the systems (8) will asymptotically stable at the origin. Besides, for the disturbance observer (6), the information of neighbors' neighbors is required due to the non-identical disturbances with unknown amplitudes and phases.

Remark 3. The formation feasibility condition (9) can be checked in a way similar to the linear multagent systems counterpart in 22 . Let $\hat{B}=\left[\bar{B}^{T}, \tilde{B}^{T}\right]^{T}$ be a nonsingular matrix with $\bar{B} \in \mathbb{R}^{m \times n}$ and $\tilde{B} \in \mathbb{R}^{(n-m) \times n}$ such that $\bar{B} B=I$ and $\tilde{B} B=0$. First step is to check the feasibility of the following condition

$$
\lim _{t \rightarrow \infty}\left(\tilde{B} A d_{i}(t)-\tilde{B} \dot{d}_{i}(t)\right)=0, \forall i=1,2, \cdots, N
$$

If (26) is satisfied, then the external command input $v_{i}(t)$ is chosen as $v_{i}(t)=-\bar{B} A d_{i}(t)+\bar{B} \dot{d}_{i}(t)$ to guarantee that

$$
\lim _{t \rightarrow \infty}\left(\bar{B} A d_{i}(t)+v_{i}(t)-\bar{B} \dot{d}_{i}(t)\right)=0 \forall i=1,2, \cdots, N .
$$

With (26) and (27), the condition (9) is guaranteed. The proof is given by Theorem 3 in 22 .

Remark 4. Note that $q_{\min }, q_{\max }, r_{1}, r_{2}$, and $r_{3}$ can be easily calculated from the Laplacian matrix of any given network connection. It is worth mentioning that the Laplacian matrix $\mathcal{L}$ is a global information. In this sense, the formation control protocols proposed in this paper are not fully distributed. The results in this paper can be extended to the fully distributed cases by following the procedures shown in $29,41-43$.

\section{Simulation}

In this section, we will demonstrate the formation control method with disturbance rejection under the leader-follower setup of five subsystems subject to the connection topology specified by the following 


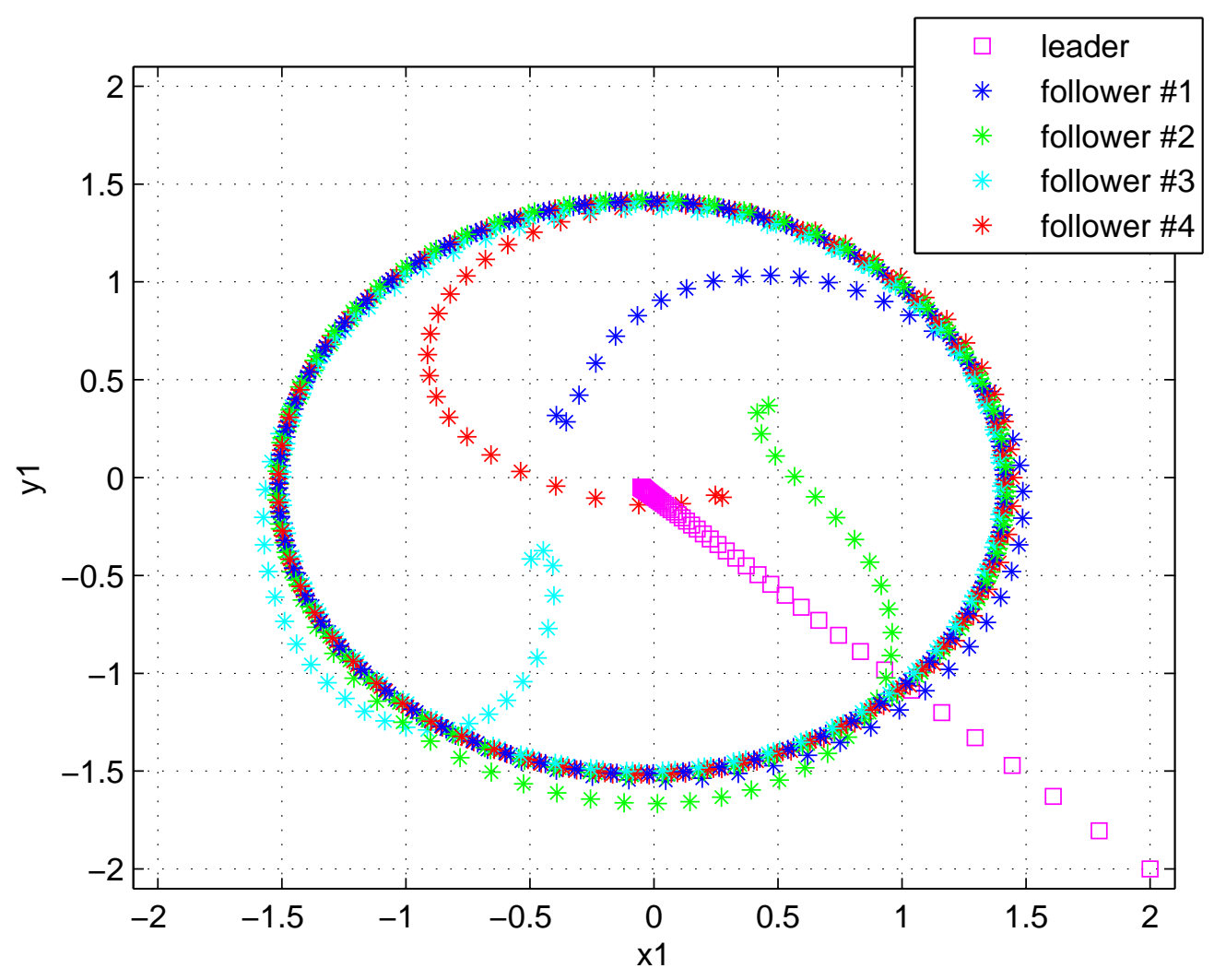

Figure 2 Trajectories of the leader and followers.

adjacency matrix

$$
\mathcal{A}=\left[\begin{array}{lllll}
0 & 0 & 0 & 0 & 0 \\
1 & 0 & 0 & 1 & 0 \\
0 & 1 & 0 & 0 & 0 \\
0 & 0 & 1 & 0 & 1 \\
1 & 1 & 0 & 0 & 0
\end{array}\right]
$$

The communication graph is shown in Figure 1, from which it shows that only the followers indexed by 1 and 4 can get access to the leader and the communication topology contains a directed spanning tree. The dynamics of the $i$ th agent are described by (1), with

$$
A=\left[\begin{array}{cc}
-1 & 1 \\
0 & 0
\end{array}\right], B=\left[\begin{array}{l}
0 \\
1
\end{array}\right], \phi\left(x_{i}\right)=g\left[\begin{array}{l}
\sin \left(x_{i 1}(t)\right) \\
\sin \left(x_{i 2}(t)\right)
\end{array}\right]
$$

In this scenario, it is supposed that external disturbances exist in the control channel. The external disturbance $w_{i}(t)$ is generated by (2) with

$$
S=\left[\begin{array}{cc}
0 & -0.1 \\
0.1 & 0
\end{array}\right], \quad F=\left[\begin{array}{ll}
1 & 1
\end{array}\right]
$$

which represents an external periodic disturbance with known frequency but without any information of its magnitude and phase. The Lipschitz constant is $\gamma=g=0.03$. It can be checked that both 


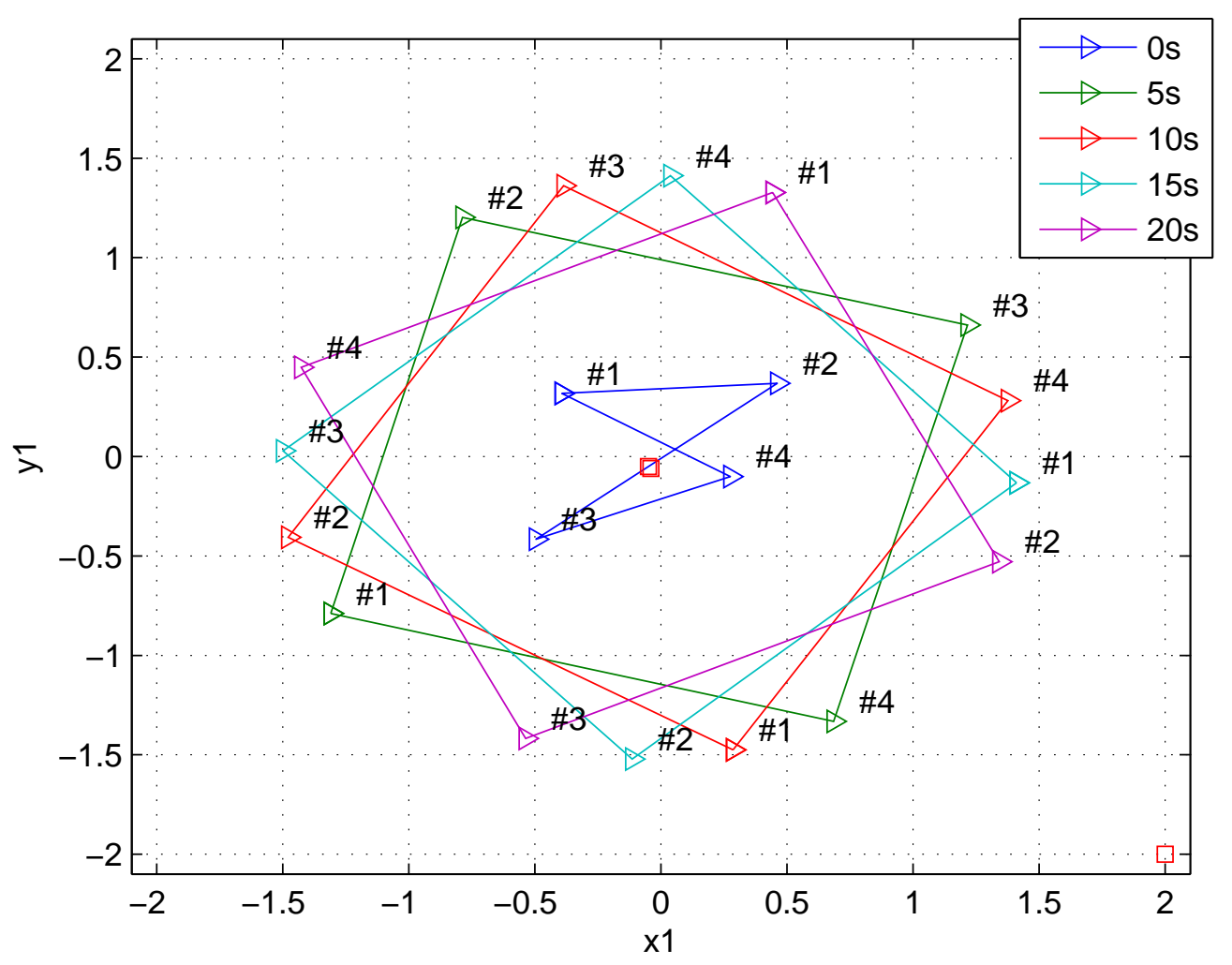

Figure 3 Trajectory snapshots at time $t=0 s, t=5 s, t=10 s, t=15 s, t=20 s$.

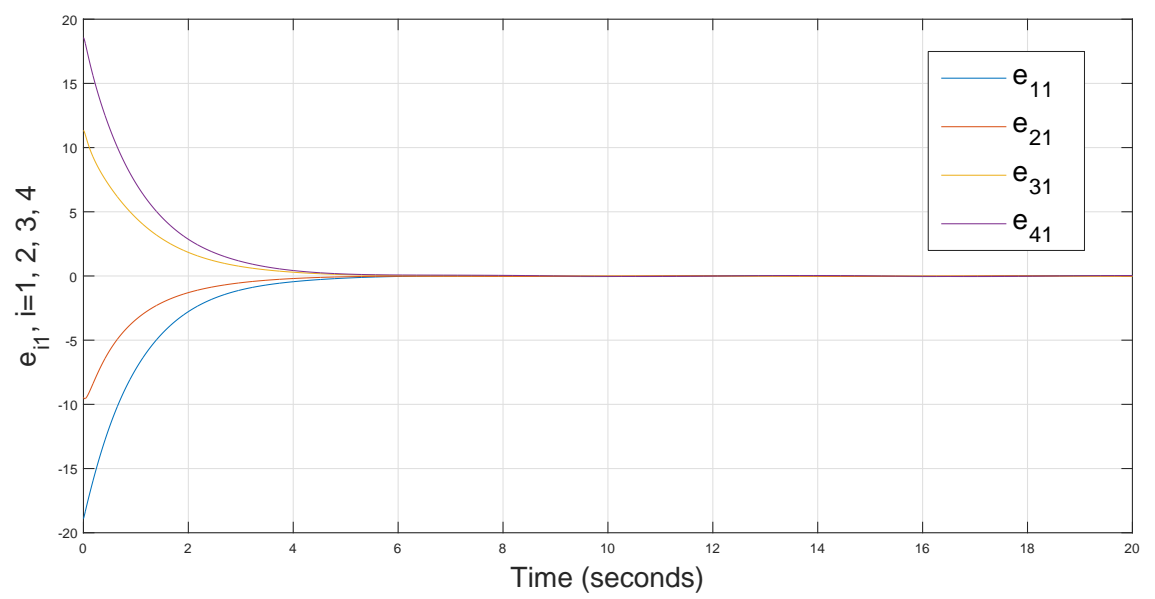

Figure 4 The formation error of state 1 with $g=0.05$. 


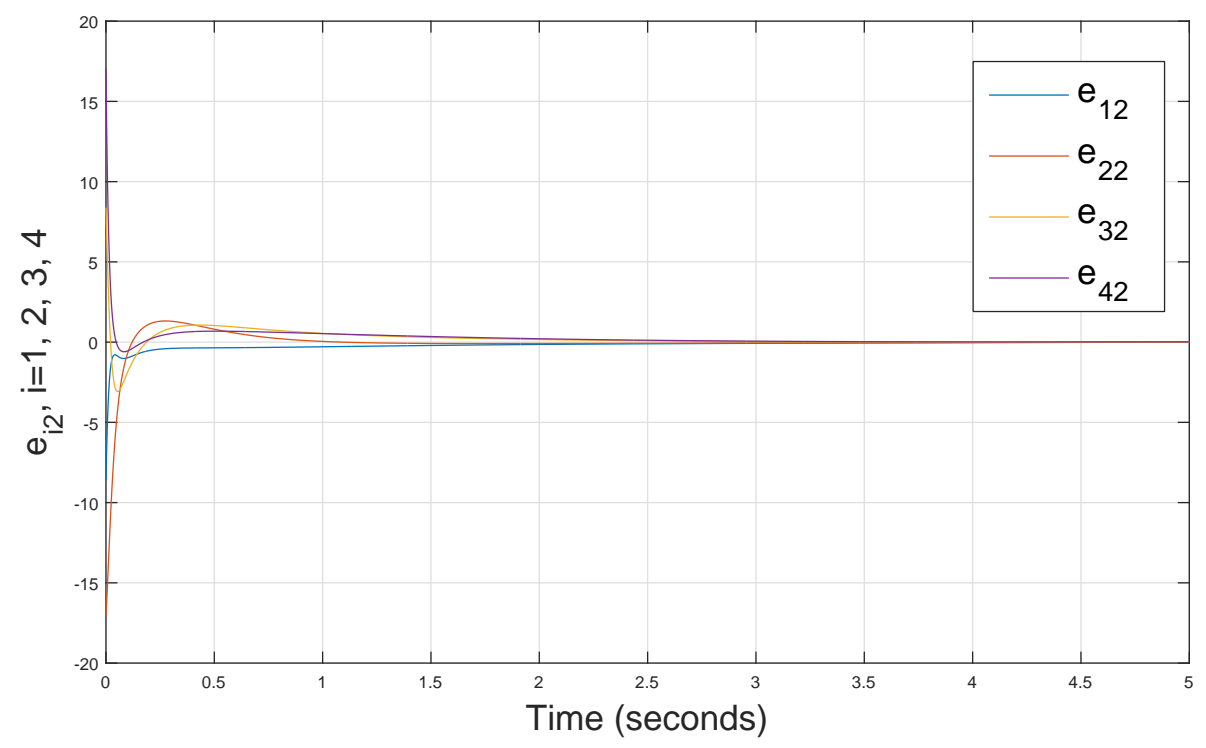

Figure 5 The formation error of state 2 with $g=0.05$.
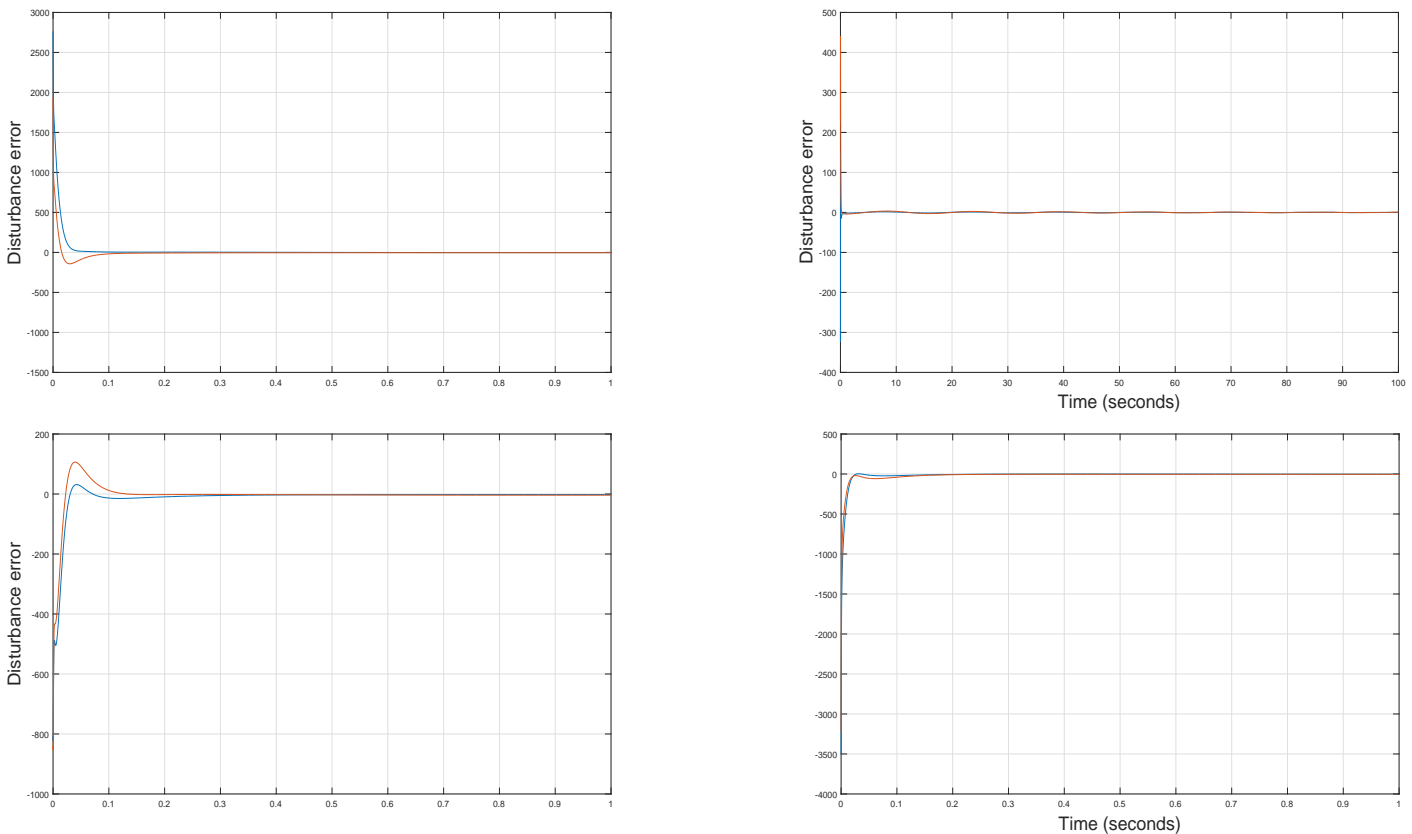

Figure 6 The estimation errors of the disturbance observers.

Assumptions 1 and 4 are satisfied. The Laplacian matrix $\mathcal{L}_{1}$ associated with $\mathcal{A}$ is that

$$
\mathcal{L}_{1}=\left[\begin{array}{cccc}
2 & 0 & -1 & 0 \\
-1 & 1 & 0 & 0 \\
0 & -1 & 2 & -1 \\
-1 & 0 & 0 & 2
\end{array}\right]
$$

Following Lemma 1, we obtain that $Q=\operatorname{diag}\left\{\begin{array}{llll}0.3846 & 0.3571 & 0.5556 & 0.7143\end{array}\right\}$ and $\rho_{0}=0.2573$. With $q_{\max }=0.7143$ and $2 q_{\max } / \rho_{0}=5.5523$, we set $c=6$ in the control input (5).

The initial states of agents are chosen randomly within $[-20,20]$. With the conditions (12) and (13), 
feasible solutions of the feedback gain $K$ and the observer gain $L$ are found to be

$$
K=[0.0333-0.6113], \quad L=\left[\begin{array}{cc}
0 & 21.6926 \\
0 & 1.6114
\end{array}\right] .
$$

The formation is defined as follows:

$$
d_{i}(t)=\left[\begin{array}{c}
\sin \left(t+\frac{(i-1) \pi}{2}\right)-\cos \left(t+\frac{(i-1) \pi}{2}\right) \\
2 \sin \left(t+\frac{(i-1) \pi}{2}\right)
\end{array}\right],
$$

which presents a periodic time-varying formation and keep rotating around the leader.

Simulation study has been carried out with different disturbances for the followers. The trajectories of five agents are shown as Figure 2 . Figure 3 shows the trajectory snapshots with $t=0 s, 5 s, 10 s, 15 s, 20 s$.

It can be seen that the time-varying formation is achieved with predefined formation reference. The formation tracking errors between the four followers and the leader are shown in Figures 4 and 5. The disturbance observation errors are shown in Figure 6. From the results shown in these figures, we can see that all the five agents reach formation although they are subject to different disturbances. Therefore, the conditions specified in Theorem 1 and (26)-(27) are sufficient to guarantee the formation disturbance rejection.

\section{Conclusion}

In this paper, the formation control problem for a class of Lipschitz nonlinear multi-agent systems with external disturbances has been addressed. Disturbance observer and time-varying formation protocol is proposed for each follower based on the relative state and formation information of the neighbouring agents. The influence of the Lipschitz nonlinearity has been taken into account in the formation stability analysis and sufficient conditions for global stability of the formation control are identified using Lyapunov method in the time domain. The proposed strategy and analysis guarantee that all signals in the closed-loop dynamics are uniformly ultimately bounded and the formation tracking errors converge to an arbitrarily small residual set. Finally, the effectiveness of the theoretical results has been illustrated by a numerical example.

Acknowledgements This work was supported by the National Natural Science Foundation of China (No. 61673034), and the China Scholarship Council (CSC).

Conflict of interest The authors declare that they have no conflict of interest.

\section{References}

1 Ren W, Beard R W. Distributed consensus in multi-vehicle cooperative control. London: Springer-Verlag, 2008.

2 Jadbabaie A, Lin J, Morse A S. Coordination of groups of mobile autonomous agents using nearest neighbour rules. IEEE Trans Autom Control, 2003, 48: 988-1001

3 Olfati-Saber R, Murray R M. Consensus problems in networks of agents with switching topology and time-delays. IEEE Trans Autom Control, 2004, 49: 1520-1533

4 Ren W, Beard R W. Consensus seeking in multiagent systems under dynamically changing interaction topologies. IEEE Trans Autom Control, 2005, 50: 655-661

5 Li Z, Duan Z, Chen G, et al. Consensus of multi-agent systems and synchronization of complex networks: a unified viewpoint. IEEE Trans Circuits Syst I, 2010, 57: 213-224

$6 \mathrm{Yu} \mathrm{W}$, Chen G, Lu J, et al. Synchronization via pinning control on general complex networks. SIAM J Contr Optim, 2013, 51: 1395-1416

7 Li Z, Ren W, Liu X, et al. Consensus of multi-agent systems with general linear and Lipschitz nonlinear dynamics using distributed adaptive protocols. IEEE Trans Autom Control, 2013, 58: 1786-1791

8 You K, Xie L. Network topology and communication data rate for consensusability of discrete-time multi-agent systems. IEEE Trans Autom Control, 2011, 56: 2262-2275

9 Wang C, Zuo Z, Lin Z, et al. A truncated prediction approach to consensus control of Lipschitz nonlinear multi-agent systems with input delay. IEEE Trans Control Netw Syst. In press. 
10 Liu S, Xie L, Lewis F L. Synchronization of multi-agent systems with delayed control input information from neighbours. Automatica, 2011, 47: 2152-2164

11 Zuo Z. Nonsingular fixed-time consensus tracking for second-order multi-agent networks. Automatica, 2015, 54: 305309

12 Qiu Z, Liu S, Xie L. Distributed constrained optimal consensus of multi-agent systems. Automatica, 2016, 68: 209-215

$13 \mathrm{Li} \mathrm{T}, \mathrm{Fu} \mathrm{M}$, Xie L, et al. Distributed consensus with limited communication data rate. IEEE Trans Autom Control, 2011, 56: 279-292

14 Ding Z, Li Z. Distributed adaptive consensus control of nonlinear output-feedback systems on directed graphs. Automatica, 2016, 72: 46-52

15 Consolini L, Morbidi F, Prattichizzo D, et al. Leader-follower formation control of nonholonomic mobile robots with input constraints. Automatica, 2008, 44: 1343-1349

16 Duan H B, Luo Q N, Yu Y X. Trophallaxis network control approach to formation flight of multiple unmanned aerial vehicles. Sci China Tech Sci, 2013, 56: 1066?1074

17 Balch T, Arkin R C. Behavior-based formation control for multirobot teams. IEEE Trans Robot Autom, 1998, 14: 926-939

18 Oh K K, Park M C, Ahn H S. A survey of multi-agent formation control. Automatica, 2015, 53: 424-440

19 Fax J A, Murray R M. Information flow and cooperative control of vehicle formations. IEEE Trans Autom Control, 2004, 49: 1465-1476

20 Ren W. Consensus strategies for cooperative control of vehicle formations. IET Control Theory Appl, 2007, 1: 505-512

21 Ren W, Sorensen N. Distributed coordination architecture for multi-robot formation control. Robot Auton Syst, 2008, 56: $324-333$

22 Dong X, Xi J, Lu G, et al. Formation control for high-order linear time-invariant multiagent systems with time delays. IEEE Trans Control Netw Syst, 2014, 1: 232-240

$23 \mathrm{Qu}$ Z. Cooperative Control of Dynamical Systems: Applications to Autonomous Vehicles. London: Springer-Verlag, 2009.

24 Zhang X, Duan H, Yu Y. Receding horizon control for multi-UAVs close formation control based on differential evolution. Sci China Inf Sci, 2010, 53: 223-235

25 Sun C, Duan H, Shi Y. Optimal satellite formation reconfiguration based on closed-loop brain storm optimization. IEEE Comput Intell Mag, 2013, 8: 39-51

26 Xie L. Output feedback $H_{\infty}$ control of systems with parameter uncertainty. Int J Control, 1996, 63: 741-750

27 Luo Y, Wang Z, Liang J, et al. $H_{\infty}$ Control for 2-D fuzzy systems with interval time-varying delays and missing measurements. IEEE Trans Cybern. In press.

28 Wang C, Ding Z. $H_{\infty}$ consensus control of multi-agent systems with input delay and directed topology. IET Control Theory Appl., 2016, 10: 617-624

29 Ding Z. Consensus disturbance rejection with disturbance observers. IEEE Trans Ind Electron, 2015, 62: 5829-5837

30 Yang J, Ding Z, Chen W H, et al. Output-based disturbance rejection control for non-linear uncertain systems with unknown frequency disturbances using an observer backstepping approach. IET Control Theory Appl., 2016, 10: $1052-1060$

31 Wu H N, Liu Z Y, Guo L. Robust $L_{\infty}$-gain fuzzy disturbance observer-based control design with adaptive bounding for a hypersonic vehicle. IEEE Trans Fuzzy Syst, 2014, 22: 1262-1271

32 Wu H N, Wang H D, Guo L. Finite dimensional disturbance observer based control for nonlinear parabolic PDE systems via output feedback. J Process Control, 2016, 48: 25-40

33 Chen W H, Yang J, Guo L, et al. Disturbance observer-based control and related methods: an overview. IEEE Trans Ind Electron, 2015, 63: 1083-1095

34 Wang C, Zuo Z, Sun J, Yang J, et al. Consensus disturbance rejection for Lipschitz nonlinear multi-agent systems with input delay: A DOBC approach. J Franklin Inst, 2017, 354: 298-315

35 Isidori A, Byrnes C I. Output regulation of nonlinear systems. IEEE Trans Autom Control, 1990, 35: 131-140

36 Ding Z. Output regulation of uncertain nonlinear systems with nonlinear exosystems. IEEE Trans Autom Control, 2006, 51: 498-503

$37 \mathrm{Su} \mathrm{Y,} \mathrm{Huang} \mathrm{J.} \mathrm{Cooperative} \mathrm{output} \mathrm{regulation} \mathrm{with} \mathrm{application} \mathrm{to} \mathrm{multi-agent} \mathrm{consensus} \mathrm{under} \mathrm{switching} \mathrm{network.}$ IEEE Trans Cybern, 2012, 42: 864-875.

38 Ding Z. Consensus output regulation of a class of heterogeneous nonlinear systems. IEEE Trans Autom Control, 2013, 58: $2648-2653$

39 Ding Z. Adaptive consensus output regulation of a class of nonlinear systems with unknown high-frequency gain. Automatica, 2015, 51: 348-355

40 Ding Z. Distributed adaptive consensus output regulation of network-connected heterogeneous unknown linear systems on directed graphs. IEEE Trans Autom Control. In press.

41 Li Z, Ren W, Liu X, et al. Distributed consensus of linear multi-agent systems with adaptive dynamic protocols. Automatica, 2013, 49: 1986-1995

42 Li Z, Ren W, Liu X, et al. Designing fully distributed consensus protocols for linear multi-agent systems with directed graphs. IEEE Trans Autom Control, 2015, 60: 1152-1157

43 Sun J, Geng Z, Lv Y, et al. Distributed adaptive consensus disturbance rejection for multi-agent systems on directed graphs. IEEE Trans Control Netw Syst. In press. 